Research Paper

\title{
Identifying Key Genes for Nasopharyngeal Carcinoma by Prioritized Consensus Differentially Expressed Genes Caused by Aberrant Methylation
}

\author{
Yunqin Chen ${ }^{1}$, Chun Zhou ${ }^{2}$, Huabin $\mathrm{Li}^{2}$, Hong $\mathrm{Li}^{3 凶}$ and Yixue $\mathrm{Li}^{1,3 凶}$ \\ 1. School of Life Sciences and Biotechnology, Shanghai Jiao Tong University, 800 Dong Chuan Road, Shanghai 200240, China \\ 2. Center for Allergic and Inflammatory Diseases \& Department of Otolaryngology, Head and Neck Surgery, Affiliated Eye, Ear, Nose and Throat Hospital, Fudan \\ University, Shanghai 200031, China. \\ 3. CAS Key Laboratory of Computational Biology, CAS-MPG Partner Institute for Computational Biology, Shanghai Institute of Nutrition and Health, Shanghai Institutes \\ for Biological Sciences, University of Chinese Academy of Sciences, Chinese Academy of Sciences, Shanghai 200031, China. \\ $\triangle$ Corresponding authors: Hong Li, E-mail: lihong01@sibs.ac.cn; Yixue Li, E-mail: yxli@sibs.ac.cn.
}

(C) The author(s). This is an open access article distributed under the terms of the Creative Commons Attribution License (https://creativecommons.org/licenses/by/4.0/). See http://ivyspring.com/terms for full terms and conditions.

Received: 2020.06.12; Accepted: 2020.10.21; Published: 2021.01.01

\begin{abstract}
Background: Nasopharyngeal carcinoma (NPC) is an Epstein-Barr virus (EBV)-associated epithelial malignancy. Large-scale genetics or epigenetics studies of NPC have been relatively scarce and sporadic, and there are no effective targeted drugs for NPC. Integrative analysis of multiple different omics profiles has been proved to be an effective approach to shed new light on cancer.

Methods: We developed a pipeline to aggregate consensus differentially expressed genes (DEGs) from multiple expression datasets from different platforms. Integrated bioinformatics analysis of DNA methylation and gene expression was used to prioritize key genes in NPC. We explored the biological and clinical importance of key genes, combining differential co-expression analysis, network analysis of protein-protein and microRNA (miRNA)-target interactions, and pan-cancer survival analysis.

Results: We obtained 668 upregulated and 594 downregulated consensus DEGs, which enriched in the PI3K-AKT, NF-KB and immune-related pathways. In NPC, $98 \%$ of 3364 differentially methylated sites were hypermethylated. Actively expressed EBV gene EBNAI was positively correlated with over-expressed genes coding DNA methyltransferase and Polycomb group proteins, suggesting that EBV infection may have an important role in the hypermethylation of NPC. Through integrated analysis of DNA methylation and mRNA and miRNA expression profiles, we prioritized 56 hypermethylated downregulated genes, including 7 tumor suppressor genes, and constructed a miRNA-target regulation network consisting of 12 hypermethylated miRNAs and 25 upregulated oncogenes. The promoter hypermethylation of PRKCB causing its downregulation was validated by experimental results and higher $P R K C B$ expression was associated with longer overall survival in head-neck squamous cell carcinoma, suggesting the potential of PRKCB as a promising disease biomarker for NPC.

Conclusions: Our integrative analysis provides reliable key genes for candidate biomarkers for diagnosis and prognosis in NPC. Based on the combined evidence of promoter hypermethylation, expression up-regulation, and association with overall survival, genes such as SCUBE2, PRKCB, IKZFI, MAP4KI, and GATA6 could be promising novel diagnostic biomarkers, and miRNAs including MIRI50, MIRI52, and MIR34 could be candidate prognosis biomarkers.
\end{abstract}

Key words: integrative analysis; differently expressed genes; EBV infection; aberrant methylation; disease biomarkers

\section{Introduction}

Nasopharyngeal carcinoma (NPC) is a malignant neoplasm that arises from the epithelium of the nasopharynx and shows remarkably skewed geographic and racial distributions. NPC is rare worldwide but common in South China, with a strikingly higher incidence [1].

NPC pathogenesis has been reported to be strongly associated with multiple factors, including 
host genetics, viral infection, and environmental effects, which can result in genetic and epigenetic alternations [2]. A recent study showed that different subtypes of NPC could be distinguished by differences in immune cell genes, and suggested that both tumor genetics and Epstein-Barr virus (EBV) infection could influence the tumor microenvironment [3]. Aberrant epigenetic alterations such as DNA methylation can disrupt or over-activate critical signaling pathways. Compared with other cancer types, such as liver, head and neck, colon, lung, thyroid, kidney, breast, pancreatic, and prostate cancers, NPC has a higher hypermethylation frequency $[4,5]$. Genes downregulated by promoter hypermethylation could represent biomarkers for disease progression and prognosis in NPC. However, transcriptome or epigenetics studies of NPC have been relatively scarce and sporadic. Compared with the use of a single dataset and single omics, integrative analysis of expression profiles and methylation profiles has proved to be an effective way to gain new insights and may shed more light on the molecular mechanism of carcinogenesis in NPC.

In this study, we developed a pipeline to aggregate consensus differentially expressed genes (DEGs) from multiple datasets from different platforms, including both microarray and RNA sequencing (RNA-seq) data, and combined methylation profiles and microRNA (miRNA) expression profiles to identify aberrantly methylated DEGs or DEGs regulated by differentially methylated (DM) miRNAs. We prioritized DEGs using voting and robust rank aggregation (RRA) [6] methods and identified reliable DEGs based on votes and RRA-adjusted p-values. As the activation of oncogenes and loss of tumor suppressor genes (TSGs) have important roles in cancer evolution, oncogenes and TSGs were investigated to select likely biomarkers and therapeutic targets for NPC. Furthermore, owing to the lack of survival information for the sample and the availability of The Cancer Genome Atlas (TCGA) data, pan-cancer analysis of the associations between gene expression and survival was also performed to determine the biological importance of these genes.

\section{Materials and Methods}

\section{Data sources for expression and methylation profiles of NPC}

We searched for "nasopharynx cancer" in EBI ArrayExpress (https://www.ebi.ac.uk/ arrayexpress/) and manually selected expression and methylation profiles of NPC. To identify biomarkers discriminating cancer from normal tissue, we studied human tissue samples using a cancer vs. cancer-free control design. Finally, six mRNA expression datasets and two methylation datasets were screened out for analysis (Table 1). To investigate differences in expression between aberrantly hypermethylated miRNAs in cancer and those in normal tissue, three miRNA expression datasets for more than 50 patients were used. Two additional datasets, GSE102349 and GSE103611, were used for interpretation or validation of our analysis results. GSE102349 contained both EBV and human expression profiles by RNA-seq from113 undifferentiated nasopharyngeal carcinoma tumors (no normal controls) and can be used to identify co-expressed EBV-host gene pairs in NPC. GSE103611 provided expression profiles for 24 NPC tumor tissues with distant metastasis after radical treatment and 24 without distant metastasis, and was used to annotate genes associated with distant metastasis.

\section{Prioritizing DEGs in NPC from multiple studies and identifying reliable consensus DEGs}

The $\mathrm{R}$ software [7] version 3.5.1 was used for data analysis. Different strategies were applied to integrate the six mRNA expression datasets from microarrays (Affymetrix and Agilent) and RNA-seq, crossing four platforms (Table 1).

Table 1. Expression and methylation datasets analyzed and integrated in the study

\begin{tabular}{|c|c|c|c|c|c|c|}
\hline Technology & Platform & Raw Dataset & \#Samplea & Design & EBV infection & DEGs dataset \\
\hline Microarray & GPL570 (HG-U133_Plus_2) & GSE34573 & $19(4+15)$ & Unpaired & EBV+ & S1 \\
\hline Microarray & GPL570 (HG-U133_Plus_2) & GSE12452 & $41(10+31)$ & Unpaired & EBV+ & \\
\hline Microarray & GPL570 (HG-U133_Plus_2) & GSE64634 & $16(4+12)$ & Unpaired & Unknown & \\
\hline Microarray & GPL96 (HG-U133A) & GSE13597 & $28(3+25)$ & Unpaired & $\mathrm{EBV}+$ & $\mathrm{S} 2 *$ \\
\hline Microarray & GPL6480 (Agilent-014850 ) & GSE53819 & $36(18+18)$ & Unpaired & Unknown & S3 \\
\hline RNA-seq & Illumina Hiseq 2000 & SRP058243 & $45(4+41)$ & Unpaired & $\mathrm{EBV}+$ & S4 \\
\hline Methylation array & Illumina HumanMethylation450 BeadChip & GSE52068 & $48(24+24)$ & Unpaired & Unknown & / \\
\hline Methylation array & Illumina HumanMethylation450 BeadChip & GSE62336 & $50(25+25)$ & Paired & Unknown & / \\
\hline miRNA array & GPL14722 & GSE32960 & $330(18+312)$ & Unpaired & EBV+ & / \\
\hline miRNA array & GPL15311 & GSE36682 & $68(6+62)$ & Unpaired & $\mathrm{EBV}+$ & / \\
\hline miRNA array & GPL20699 & GSE70970 & $264(17+246)$ & Unpaired & $\mathrm{EBV}+$ & / \\
\hline
\end{tabular}

a Sample: n (normal + cancer); ${ }^{*}$ for enough power to calculate DEGs, S2 included GSE13597 and S1. 
Of the four Affymetrix datasets, three using the sample platform GPL570 were combined into one integrated dataset S1. S2 planned to be only GSE13597, which had only 3 normal and 25 cancer samples, and used the platform GPL96. However, to achieve sufficient power to get more reliable DEGs, we merged S1 and GSE13597 into S2 (Supplementary Figure 1A). During data integration, the merged dataset was normalized and batch effects were removed using the ComBat function from 'sva' package [8] (Supplementary Figure 1B). S1 contained 20188 genes and 76 samples (18 controls and 58 NPC), and S2 contained 12403 genes and 104 samples (21 controls and 83 NPC).

S1, S2, S3 (Agilent), and S4 (RNA-seq) were used for DEGs identification. DEGs from microarrays were identified using the "limma" package [9], whereas those from RNA-seq were called using the "DESeq2" package [10]. We used STAR [11] to map RNA-seq reads to reference genome hg19. $p$-values were adjusted using the Benjamini-Hochberg $(\mathrm{BH})$ method [12]. The criteria for DEGs were: 1 ) adjusted $p<0.05 ; 2$ ) absolute fold change $>2$ between cancer and normal tissue.

Meta-analysis was used to aggregate the DEGs from each dataset. We aggregated upregulated and downregulated DEGs, respectively, using two methods: voting and RRA [6]. The vote number is the number of datasets in which genes are significantly differentially expressed. As detectable genes were not the same across the four platforms, RRA was used to pick out DEGs that were markedly changed according to only one study but not detected in other studies as data were not available. RRA scores are negatively correlated with vote number (Spearman's rho $=-0.7$ for upregulated genes and -0.55 for downregulated genes, $p<0.01$ ). A DEG with vote number $\geq 2$ or RRA $\mathrm{BH}$-adjusted $p<0.01$ was considered reliable and used for further analysis.

\section{Differential co-expression analysis of DEGs}

DEG expression profiles from S1 were used for the differential co-expression analysis, because the gene number of S1 was largest among the 3 datasets with \#normal sample $>10$. R package DCGL [13] was used to find differentially co-expressed gene pairs among the DEGs. Differential co-expression profile and differential co-expression enrichment methods were used to identify differentially co-expressed genes (DCGs) and differentially co-expressed links (DCLs). DCLs whose absolute correlation coefficients were not less than 0.5 in at least one situation (normal or cancer) were selected. To check types of link change, we classified DCLs into three types: "loss-ofassociation", "gain-of-association", and "reverse-of- association". If the absolute correlation coefficient of a DCL in cancer was significantly stronger than that in the normal condition, it would be grouped into the "gain-of-association" type, if the reverse was true, it was considered to belong to the "loss-of-association" type. The "reverse-of-association" type was the case where the direction of the relationship of DCL switched between cancer and the normal condition.

\section{DEGs probably regulated by aberrant methylated promoters or miRNAs}

For we didn't have matched samples with both expression and methylation profiles, we identify DEGs likely caused by aberrant Methylation through applying the strategy of "overlapping'. The "limma" package [9] was used to identify DM probes (DMPs). Both the methylation datasets used Illumina HumanMethylation450 BeadChip, but GSE62336 had matched normal controls, for which paired comparison design was appropriate, whereas GSE52068 used pooled normal controls and so pooled comparison design was preferable. DMPs were selected using the following cutoff: 1 ) adjusted $p<0.05$; 2 ) absolute $\beta$ change $\geq 0.2$ in one dataset and $>0.1$ in another. DMPs were annotated using the package IlluminaHumanMethylation450kanno.ilmn12.hg19

[14]. The promoter region was defined as $1500 \mathrm{bp}$ before the TSS of each gene plus $5^{\prime}$ UTR. If the promoter region of a gene or miRNA had DMPs, we considered it to be a DMG or DM miRNA. Recurrent DMGs or DM miRNAs (identified in both datasets and with the same direction of change) were used to overlap with consensus DEGs to identify DEGs likely caused by aberrant methylation (Figure 1B).

Hypomethylated upregulated or hypermethylated downregulated DEGs and over-expressed DEGs targeted by hypermethylated miRNAs or under-expressed DEGs targeted by hypomethylated miRNAs were considered to be aberrant methylationregulated DEGs.

\section{Gene function enrichment analysis, transcription factors, and cancer gene annotation}

We used the $\mathrm{R}$ package clusterProfiler [15] to perform gene ontology and pathway enrichment analysis. Adjusted $p$-values less than 0.01 were obtained by the $\mathrm{BH}$ method were regarded as statistically significant. The human transcription factor list was downloaded from the supplementary file of Lambert et al. [16].

The oncogene list was obtained from the ONGene database [17] (http://ongene.bioinfominzhao.org/), and the tumor suppressor genes (TSGs) list was from the TSGene database [18] 
(https://bioinfo.uth.edu/TSGene/index.html). The activation of oncogenes and inactivation of TSGs may be associated with the cancer development.

\section{Protein-Protein Interaction (PPI) and miRNA-target interactions (MTIs)}

PICKLE (Protein InteraCtion KnowLedgebasE) is a meta-database for the human direct proteinprotein interactome, integrating publicly available PPI databases via genetic information ontology [19]. A total of 179738 standard and cross-checked PPIs were downloaded from PICKLE. miRNA-target interactions (MTIs) from the experimentally validated MTI database miRTarBase are validated experimentally by reporter assay, western blot, microarray and next-generation sequencing experiments [20]. A total of 502652 human MTIs (containing 2599 miRNAs and 15050 target genes) were obtained from miRTarBase.

\section{Survival analysis of hypermethylated downregulated genes and miRNAs using TCGA data}

TCGA PanCanAtlas gene and miRNA expression and patient survival information were obtained from the NCI Genomic Data Commons Data Portal (https://gdc.cancer.gov/about-data/ publications/pancanatlas). For tumor versus normal tissue comparisons, we only considered cancer types with more than 10 normal samples. The Cox regression model and log-rank test were used to determine prognostic power.

\section{DAC treatment, RNA isolation and qRT-PCR}

Human nasal epithelial cells (hNEPC) and NPC cell line C666-1 were planted to 6-well-plate, after growing for 24 hours, the cells were treated with vehicle (DMSO) or $10 \mathrm{mM} \mathrm{DAC} \mathrm{(5-aza-2'-deoxy-}$ cytidine, MCE NSC 127716). The medium containing DMSO/DAC were changed every 24 hours for 72 hours. After treatment, total RNAs were extracted using TRIzol (Invitrogen Cat No.:10296010) according to the manufacturer's instruction. cDNA was reversely transcribed from $500 \mathrm{ng}$ total RNA by PrimeScript RT-PCR kit (Takara Cat No.: RR014). Real-time PCR was carried out on an ABI 7900HT Fast Real-Time PCR System. Data shown were the relative abundance of the indicated mRNA normalized to that of Gapdh by the change-in-cycling-threshold $(\Delta \Delta \mathrm{CT})$ method. The primers for real-time PCR were listed in the Supplementary Table 1.

\section{Results}

\section{Identification and Characteristics of consensus DEGs}

We collected all available qualified expression and methylation profiles for NPC including six mRNA expression datasets (Table 1). To integrate mRNA expression profiles from different studies and platforms we first merged datasets from the same platform by unique gene ID and removed batch effects using the ComBat method [21]. After merging, four datasets from four platforms were used to determine DEGs between NPC and normal tissues. The analysis pipeline for expression and methylation data is illustrated in Figure 1.

In total, 2670 upregulated genes and 2217 downregulated genes were identified at least one dataset (Figure 2A). Then we used meta-analysis to integrate DEGs from different platforms, using a combination of vote number and RRA to obtain consensus DEGs (see Materials and Methods). If a DEG had vote number $\geq 2$ or RRA-adjusted $p<0.01$, it was considered to be reliable and used for further analysis. Finally, 1261 DEGs were obtained, including 668 upregulated and 594 downregulated genes (gene S100A2 was downregulated in S3 and S4 but overexpressed in S1 and S2). Functional enrichment analysis of all DEGs revealed that they were enriched in cancer-related pathways including the PI3K-AKT signaling pathway, NF-kB signaling pathway, p53 signaling pathway, focal adhesion and immunerelated pathways, chemokine signaling pathway, IL-17 signaling pathway, and B cell receptor signaling pathway (Figure 2B). Virus-associated pathways (Kaposi sarcoma-associated herpesvirus infection and human papillomavirus infection) were also overrepresented.

To further understand the functions of DEGs, we annotated DEGs with oncogenes, TSGs, and transcription factors, and ranked them by vote number and RRA score (Supplementary Table 2; see Materials and Methods). Among the 1261 DEGs, there were 60 over-expressed oncogenes and 52 downregulated TSGs. Oncogenes CXCL3, EPCAM, GATA6, NOV, and SOX4 were upregulated in all four datasets (Figure 2C), indicating their important roles in NPC carcinogenesis. LHX2 was the top upregulated gene based on RRA score. LHX2 is a transcription factor and a mesenchymal marker of epithelialmesenchymal transition (EMT), which has been reported to promote tumor growth and metastasis in pancreatic ductal adenocarcinoma and breast cancer $[22,23]$. The top three downregulated genes were ELL3, GSTA3, and MUC16 (Figure 2C). Both GSTA3 and MUC16 have protective roles against EMT [24, 
25]. Additionally, through integrating the dataset with primary and metastatic NPC (GSE103611), we identified 11 up-regulated DEGs (including oncogenes like SOX4, FNDC3B and JUP, and EMT regulatory factors like SNAI2) and 2 down-regulated DEGs (immune-related genes CR2 and LAT2) associated with distinct metastasis (Supplementary Table 2).

Next, we constructed co-expression networks of DEGs using the expression profiles in NPC and normal samples, respectively (see Materials and Methods). Differential co-expression analysis was performed to investigate changes in gene-gene links; 388 DCGs and 33509 DCLs were identified. Our results showed that almost $96.3 \%$ DCLs lost associations, $3.2 \%$ gained new associations, and only $0.4 \%$ showed reversed associations (Supplementary Figure 2A). On the other hand, 288 (74.2\%) DCGs were downregulated in NPC, significantly more than in the background (47\% DEGs were downregulated, chi-square test $\mathrm{p}<0.01)$. These results indicate that the co-expression network of NPC has a trend of loss-of-association of downregulated genes, which may result in the dysregulation of normal gene links, especially silencing of TSG regulation. For example, TSG PAX5 is a B cell transcription factor, which is downregulated in NPC. PAX5 restoration can cause rapid repression of Myc and DNA replication factor [26]. In our study, 95\% of 227 DCLs of PAX5 were of the loss-of-association type, and $83 \%$ differentially co-expressed partners (DCPs) were upregulated in NPC, probably owing to loss-of-regulation of PAX5 (Figure 2D and Supplementary Figure 2B). Functional enrichment analysis suggested that PAX5 DCPs were enriched in the cell division biological function and the DNA replication signaling pathway.

\section{Global hypermethylation and association with EBV gene expression}

EBV is an important risk factor for NPC. EBV infection can cause epigenetic changes in the host genome and promote tumorigenesis. Previous studies have shown that EBV-associated tumors are globally hypermethylated. Here, we identified 3364 DM sites, and 3284 (98\%) were hypermethylated (Figure 1B). Therefore, we tried to explore the molecular mechanism between EBV and hypermethylation. DNA methyltransferase and the Polycomb group (PcG) family proteins are critical to DNA methylation levels and often cooperate in silencing gene expression. We found that genes coding DNA methyltransferase (DNMT1, DNMT3A, DNMT3B) and PCG proteins (PRC1, EZH2, SUZ12) were overexpressed in NPC (Figure 3A). Analysis of RNA-seq

A

A S1:GSE34573

GSE12452, GSE64634 S2: S1, GSE13597

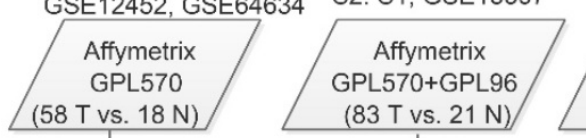

S3.

S4: SRP058243

B

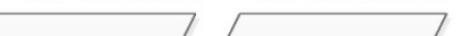

Agilent

(18 T vs. $18 \mathrm{~N})$

RNA-Seq

$(41$ T vs. $3 \mathrm{~N})$

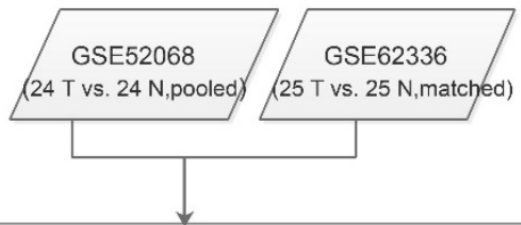

Identify DMPs that have similar change in two datasets (adjusted $P<0.05$; absolute $\beta$ change $>=0.2$ in one dataset and $>0.1$ in another dataset) (adjusted $\mathrm{P}<0.05$; absolute fold change $>2$ )
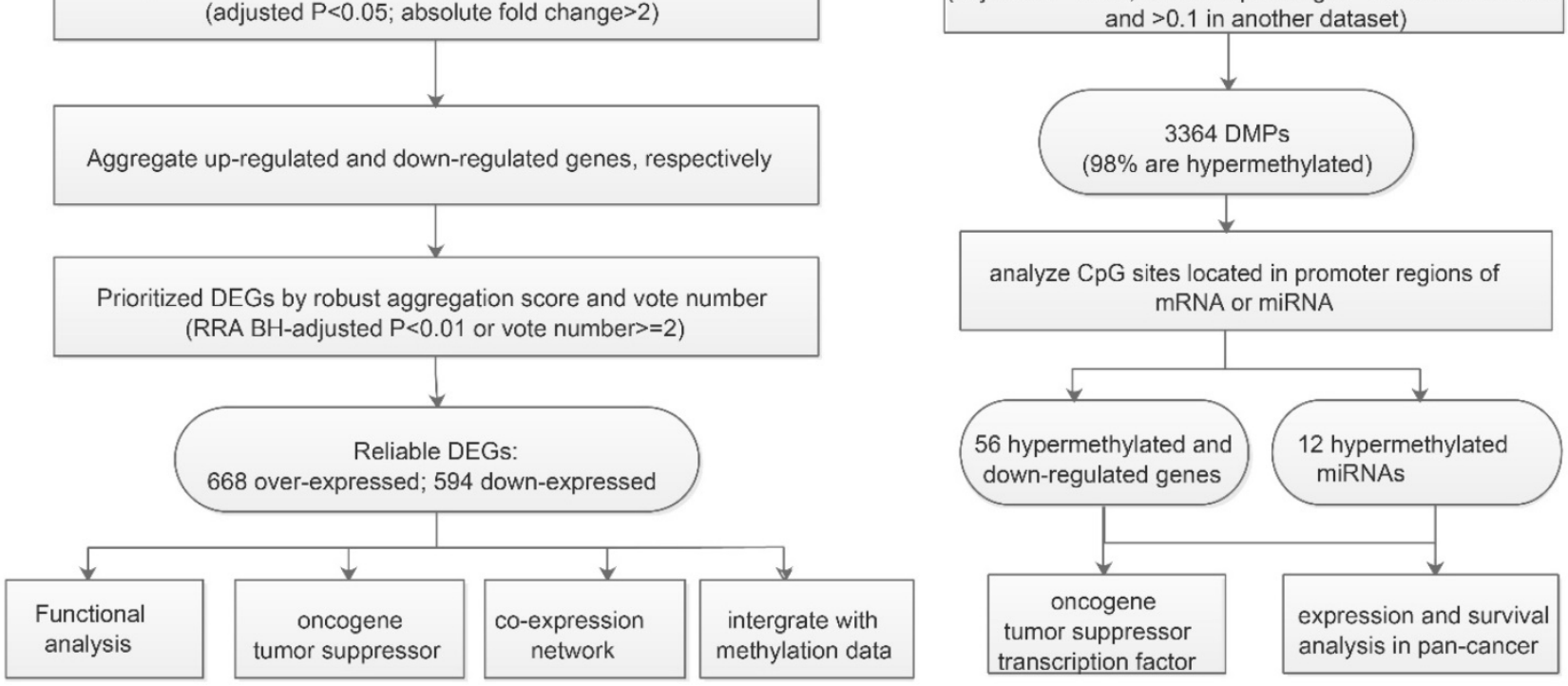

Figure 1. Workflow for identifying prioritized DEGs caused by aberrant methylation. A. Integrated analysis of multiple expression profiles to prioritize DEGs. B. Pipeline for identifying aberrantly methylated DEGs and DM miRNAs-DEGs. 
dataset S4, which contained both host and EBV transcriptomes, showed that seven lytic genes (BALF3, BALF4, BALF5, BILF1, LF1, BARF1, LF2), two latent genes (LMP-1,LMP-2B), and EBNA-1 were actively expressed in NPC patients. The lytic EBV gene products may directly induce DNA damage and contribute to NPC development. To explore whether EBV gene expression programs were associated with host gene expression of DNA methyltransferase and PcG proteins, we performed a correlation analysis between human genes and the top 10 highly expressed EBV genes. The significantly positively correlated pairs are shown in Figure 3B (Pearson's correlation coefficient $>0.3, p<0.05)$. EBNA1 was positively associated with both kinds of genes, which was confirmed by other study using dataset
GSE102349 [3] (Supplementary Table 3). EBNA1 is the only nuclear EBV protein expressed in both latent and lytic modes of infection, and is required for the replication and maintenance of the episomal EBV genome. EBNA1 is significantly correlated with $E Z H 2$, SUZ12, and DNMT3B, indicating a potential role for EBNA1 in the global hypermethylation of NPC.

\section{Downregulated genes induced by promoter hypermethylation}

Hypermethylation in gene promoters is a well-known mechanism for the silencing of TSGs. We found 56 hypermethylated and downregulated genes in NPC (Supplementary Table 2). PPI data from PICKLE were used to investigate the interactions of these 56 genes and other genes. Functional
A
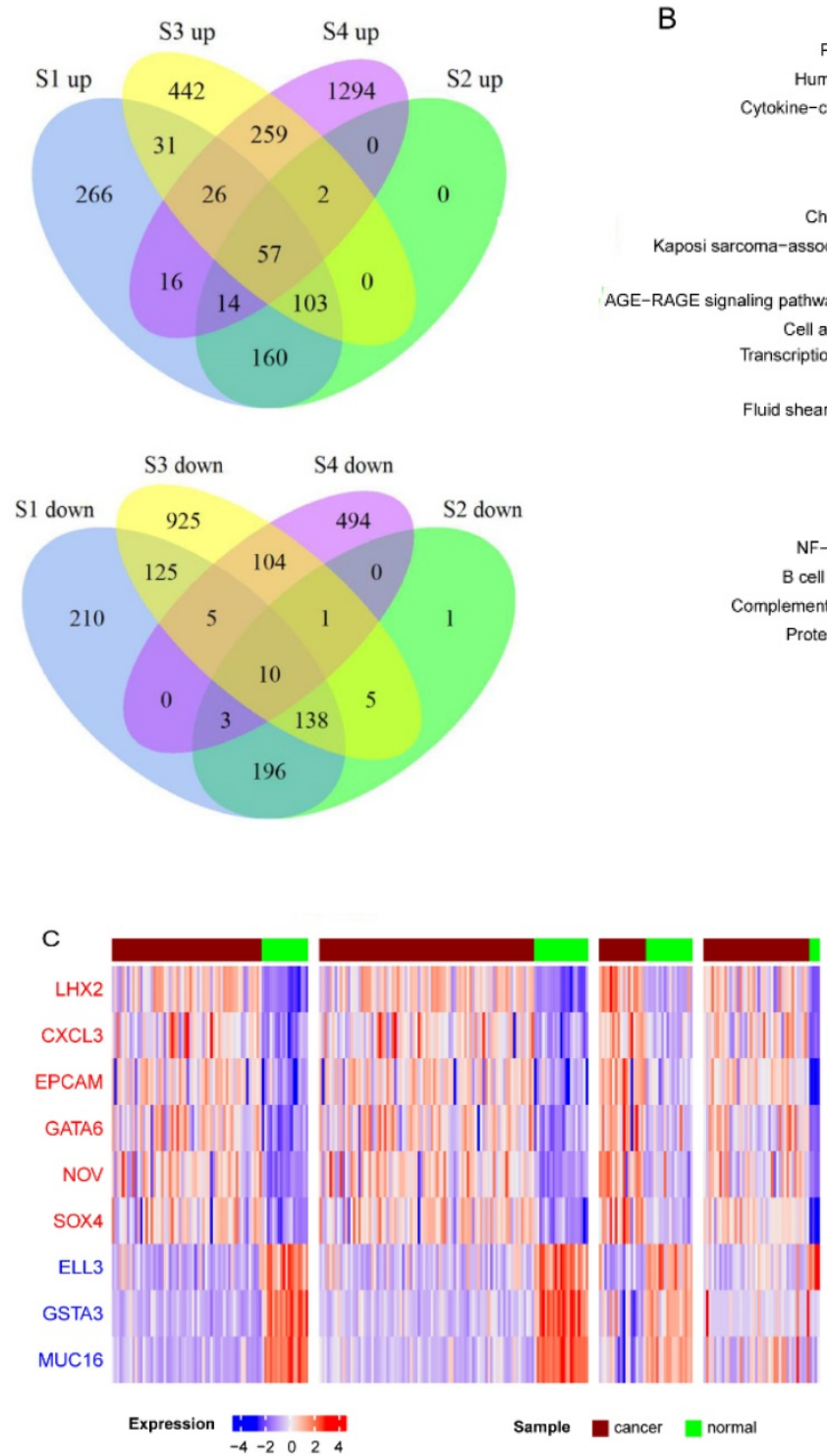

B
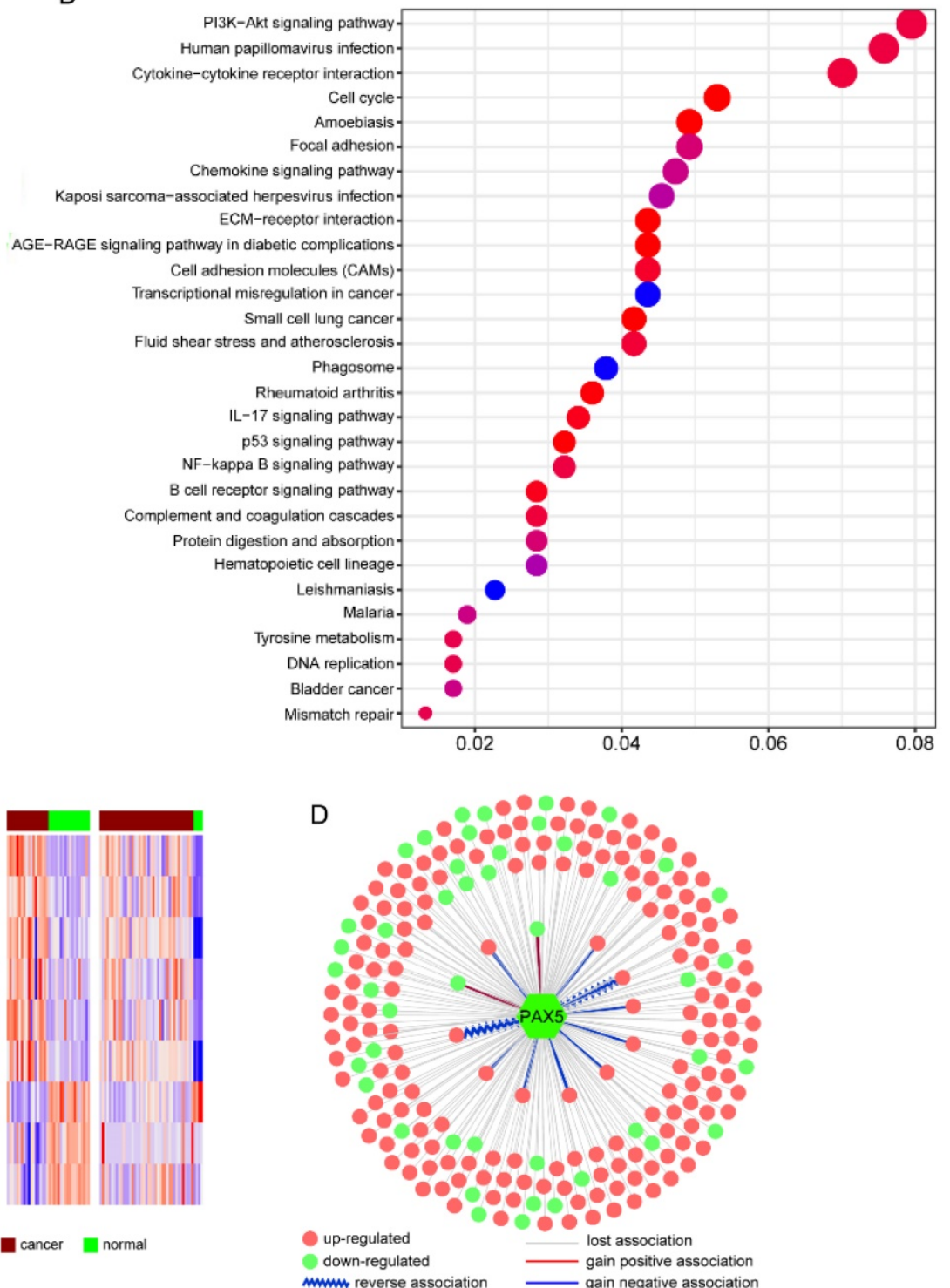

Figure 2. Characteristics of consensus DEGs. A. Venn diagram of DEGs identified from each dataset; “up” means upregulated DEGs, "down” means downregulated DEGs. B. Functional enrichment of consensus DEGs. C. Heatmap of top-ranked upregulated and downregulated DEGs. D. DCLs of PAX5 in cancer. 
enrichment analysis of all genes in the PPI network showed that they were enriched in the following immune-related pathways: "T cell receptor signaling pathway", "Natural killer cell mediated cytotoxicity", "Epstein-Barr virus infection", and "TNF signaling pathway" (Supplementary Table 4).

Table 2. Seven downregulated hypermethylated tumor suppressor genes in NPC

\begin{tabular}{lllll}
\hline Gene & Transcriptional factor & TF-domain & EMT-related & Comments* \\
\hline SCUBE2 & NO & - & Yes & Novel \\
HOPX & YES & Homeodomain & - & Known \\
IRF8 & YES & IRF & Yes & Known \\
PRKCB & YES & Unknown & - & Novel \\
IKZF1 & YES & C2H2 ZF & - & Novel \\
$M A P 4 K 1$ & NO & - & - & Novel \\
SHISA3 & NO & - & - & Known \\
\hline
\end{tabular}

*Novel: not reported in previous study. Known: validated by other studies.

Among the 56 hypermethylated and downregulated genes, there were seven TSGs (Table 2). These silenced TSGs are important for the carcinogenesis of NPC. Previous studies have reported that hypermethylation of HOPX, IRF8, and SHISA3 caused downregulation of gene expression and promoted the metastasis of NPC [27-29]. Although the roles of another four genes (SCUBE2, $P R K C B$, IKZF1, and MAP4K1) in NPC were not known, their functions were reported in other cancer types. SCUBE2 is silenced by CpG island hypermethylation in breast cancer, and its activation could inhibit cancer cell migration and invasion through the reversal of EMT [30]. Transcription factor IKZF1 is a

A
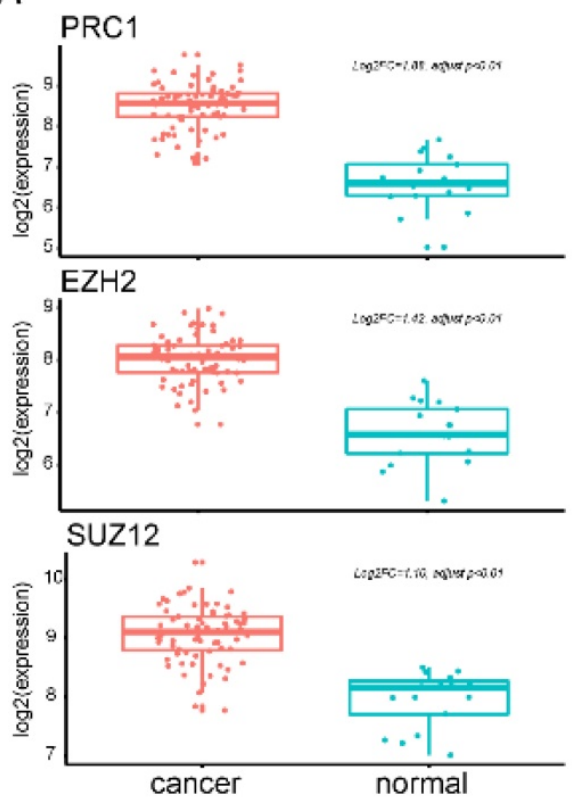

DNMT1

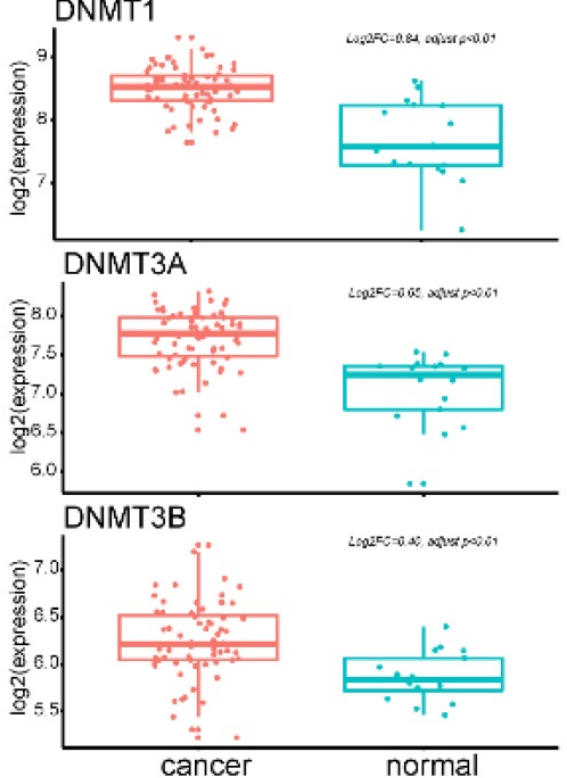

critical regulator of lymphoid differentiation and its encoding protein Ikaros regulates the development and function of the immune system [31]. PRKCB and $M A P 4 K 1$ are kinases and participate in many signaling pathways.

To better understand the biological role of the 56 hypermethylated and downregulated genes in cancer, we used data from TCGA to investigate their expression changes and relationship with survival (Figure 4A). We found that most of the 56 genes were downregulated in other cancers, especially in headneck squamous cell carcinoma (HNSC). SCARA5, MAOB, MAP6, SHISA3, USP44, CH25H, TOX, and $P R K C B$ were downregulated in at least 10 types of cancer. Expression of some genes tended to positively correlate with overall survival (hazard ratio $<1$, $p<0.05)$. The pan cancer analysis indicates the potential functional roles of these genes in NPC.

$P R K C B$ is both tumor suppressor gene and transcriptional factor and higher $P R K C B$ expression was associated with longer overall survival in HNSC (Figure 4B). To validate the relationship between expression of gene and methylation of its promoter in NPC, we examined the expression levels of $P R K C B$ before and after treatment with the demethylating drug DAC using human nasal epithelial cells (hNEpC) and NPC cell line C666-1 (see Methods). qRT-PCR revealed that the mRNA level of $P R K C B$ was significantly lower in NPC cell line C666-1 than hNEPC. After DAC treatment, the expression level of $P R K C B$ statistically increased in C666-1 $(p<0.001)$ (Figure 4C), which suggested $P R K C B$ downregulation in NPC was due to its promoter hypermethylation.

B

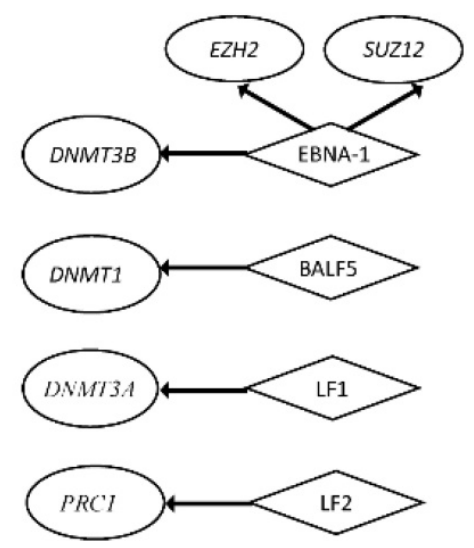

Figure 3. Ectopic expression of hypermethylation-associated genes and their association with EBV infection. A. Upregulated genes of DNA methyltransferase and PcG proteins in NPC. B. Significantly positively correlated gene pairs between actively expressed EBV genes and epigenetic regulator genes. Human epigenetic regulator genes were italicized and shown in ellipse while EBV genes were not italicized and shown in diamond. 
A.

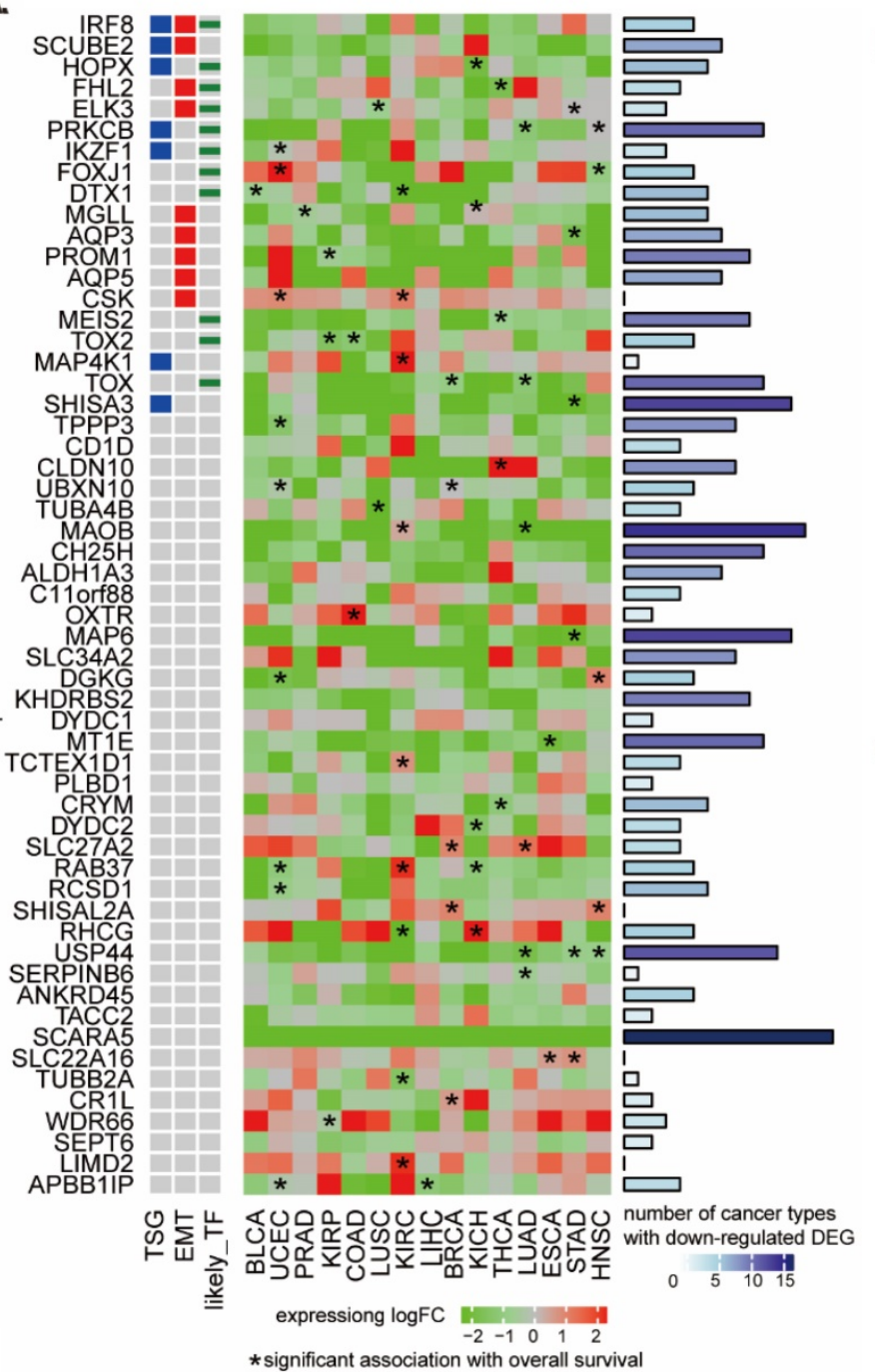

B

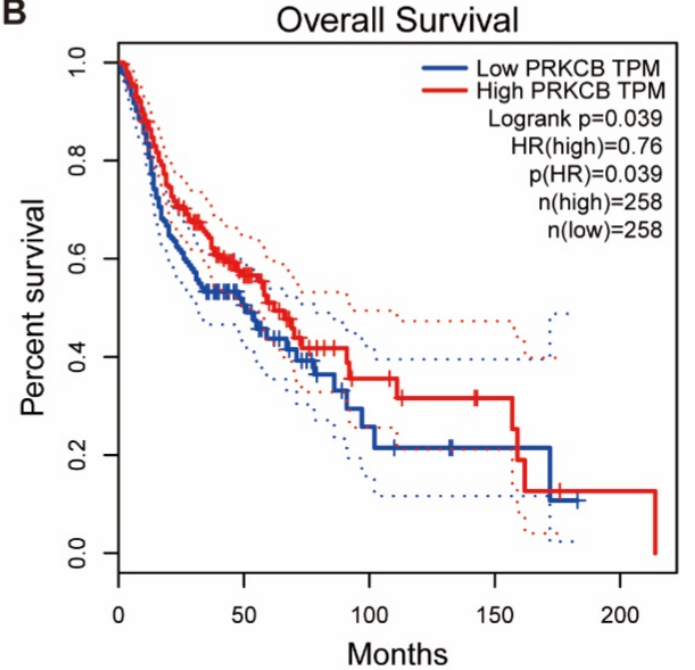

C

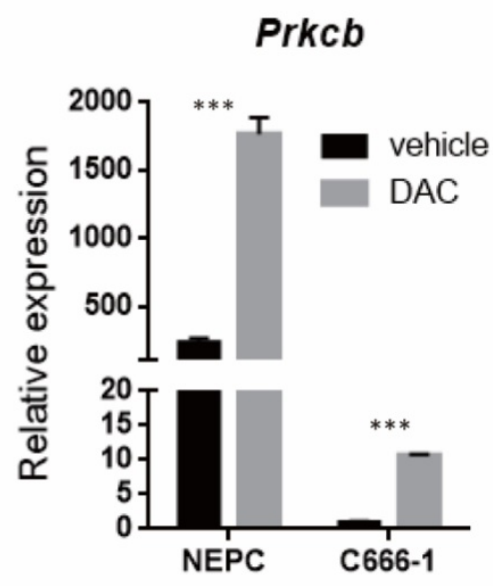

Figure 4. Pan-cancer expression and survival analysis of $\mathbf{5 6}$ hypermethylated downregulated genes. A. Pan-cancer expression and survival analysis of 56 hypermethylated downregulated genes. B. Higher PRKCB expression was associated with longer overall survival in HNSC. C. PRKCB down-expressed in NPC cell line and PRKCB expression was upregulated after DAC treatment.

\section{Oncogenes regulated by hypermethylated downregulated miRNAs through MTIs}

We found twelve miRNAs whose promoters were hypermethylated in NPC in both methylation datasets. To check whether hypermethylation in the miRNA promoters could lead to decreased expression, we analyzed three miRNA expression datasets with more than 50 patients: GSE32960, GSE36682, and GSE70970 (Table 1). Eight of the twelve hypermethylated miRNAs showed significantly lower expression in NPC than in normal tissue in at least one of the expression datasets (Supplementary Table 5).

Pan-cancer analysis showed that MIR129-2, MIR149, MIR152, MIR150, MIR34B, and MIR34C were downregulated in at least four types of cancer, and MIR150 had a protective role against death in most types of cancer (Figure 5A). Seven miRNAs
(MIR129-2, MIR149, MIR152, MIR137, MIR150, MIR34B, and MIR34C) have been reported as tumor suppressors in many types of cancer. Upregulation of the miR-34 family resulted in apoptosis and cell-cycle arrest through targeting p53 [32, 33]. MIR152 and MIR137 inhibited cell proliferation, migration, and invasion in human cancers [34-37]. Downregulation of MIR129-2 by promoter hypermethylation induced breast cancer cell proliferation and apoptosis [38]. MIR149 plays an important part in tumorigenesis and tumor progression, and its downregulation promoted the metastatic dissemination of tumor cells by supporting aberrant Rac activation in breast cancer [39]. Survival analysis of 312 NPC patients from GSE32960 showed that patients with higher MIR150, MIR137, or MIR152 had a better survival rate (log rank test, $p<0.05$, data not shown), suggesting that they also act as tumor suppressors in NPC. 


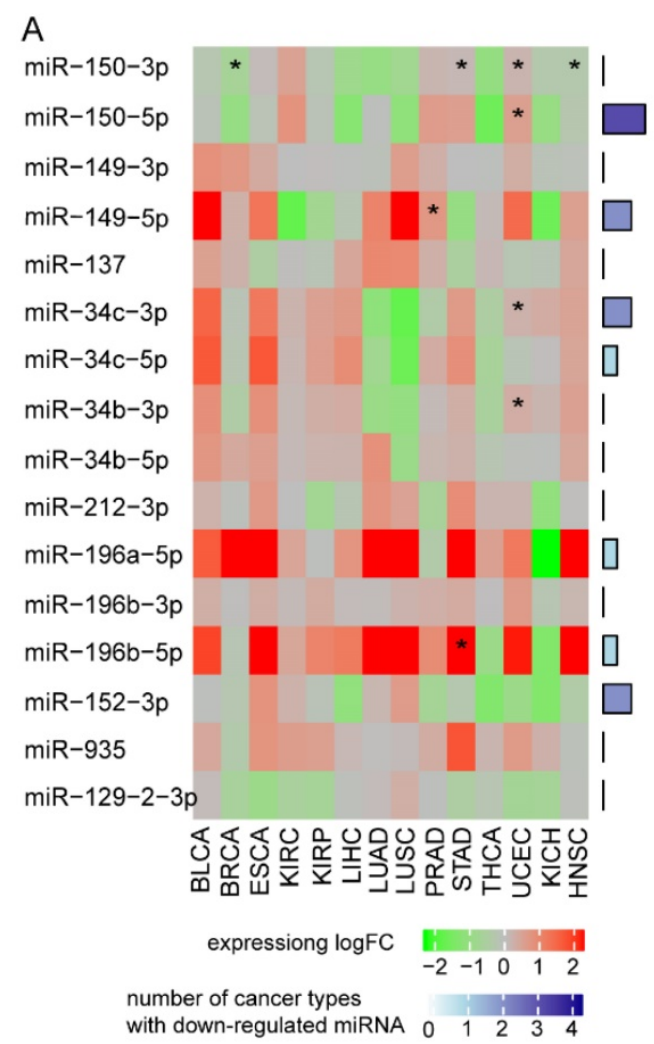

B

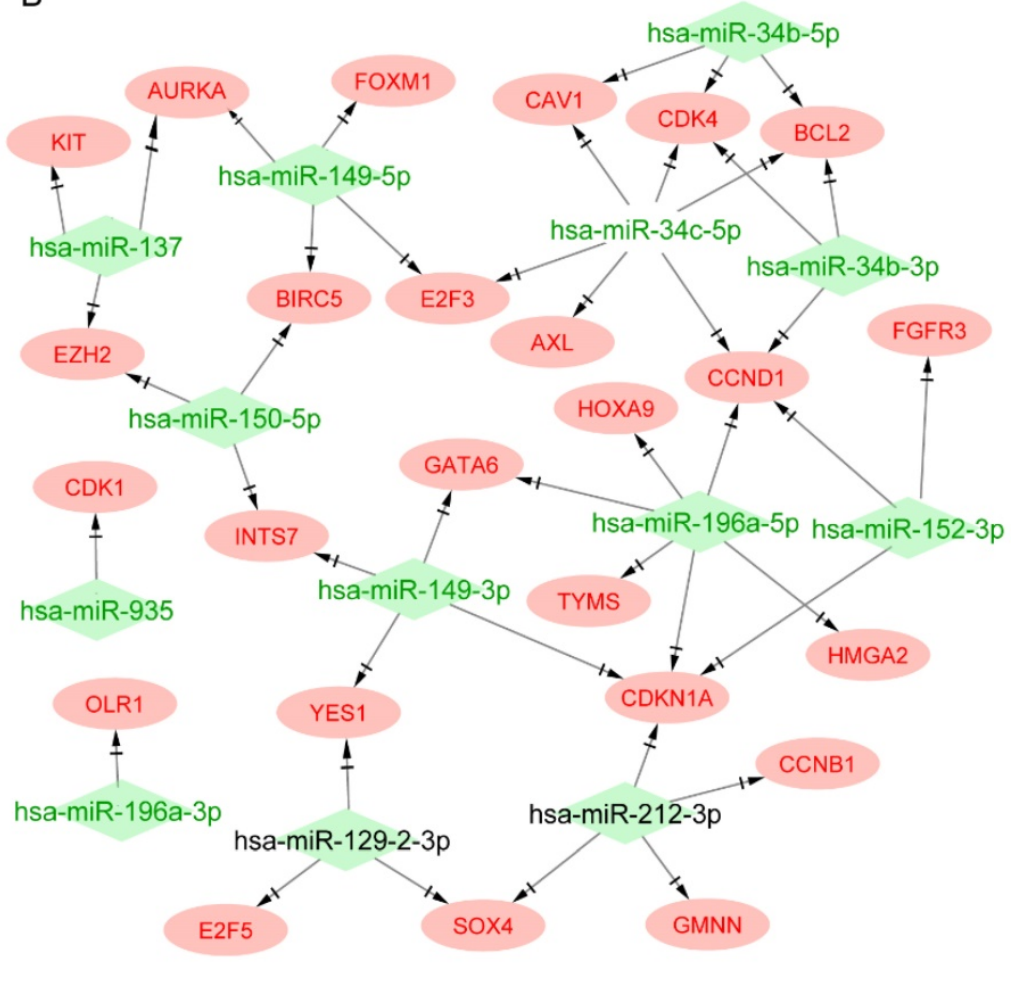

Figure 5. Oncogenes regulated by hypermethylated downregulated miRNAs through MTIs. A. Pan-cancer expression and survival analysis of 12 hypermethylated miRNAs. B. Regulation network of hypermethylated miRNAs and their targeted upregulated oncogenes.

Next, we explored the effects of 12 hypermethylated miRNAs on their target genes. As miRNAs generally repress gene expression, our hypothesis was that hypermethylation of a miRNA promoter would reduce its expression and thus increase the expression of its target genes. We found 181 MTIs between the 12 miRNAs and 128 over-expressed target genes (Supplementary Table 6). Functional analysis of 128 target genes showed enrichment in the following cancer signaling pathways: "cell cycle", "PI3K-Akt signaling pathway", and "p53 signaling pathway". Interestingly, PDL1 was highly expressed in NPC and regulated by hypermethylated MIR152 and MIR34b, indicating the potential for immunotherapy using agents mimicking miR-152 or miR-34 to inhibit PD-L1 and enhance $\mathrm{T}$ cell proliferation and effector cytokines.

Among the 128 over-expressed target genes, 25 were oncogenes, forming a miRNA-oncogene network with 43 links (Figure 5B). Several oncogenes were regulated by multiple miRNAs. Oncogenes CCND1 and CDKN1A and transcription factor Homeobox C8 (HOXC8) were regulated by four miRNAs. Ectopic expression of $\mathrm{HOXC} 8$ can modulate NPC cell growth in vitro and in vivo, and EBV gene LMP1 represses HOXC8 [40]. Epigenetic regulator $E Z H 2$ was regulated by tumor suppressor MIR150 and MIR137. Transcription factor SOX4 was overexpressed in all expression datasets and regulated by MIR129 and MIR212. The expression of MIR212 has been reported to be decreased in NPC tissues and cells, and its overexpression could inhibit the metastasis of nasopharyngeal carcinoma by targeting SOX4 [41]; patients with higher expression levels of SOX4 had poorer survival rates [42].

\section{Discussion}

NPC is poorly understood at the genetic level, and there is an urgent need for more efficient approaches such as targeted therapy and immunotherapy. There have been few studies of the genomic changes in NPC. Lin et al. in 2014 found that enrichment of genetic lesions in NPC affected several important cellular processes and pathways, including chromatin modification, ERBB-PI3K signaling, and autophagy machinery [43]. Li et al. in 2017 also identified NF-кB pathway-activating mutations during cancer pathogenesis [44]. Different subtypes of NPC harbored different genomic alterations, and a high frequency of IHD2 mutations was detected in undifferentiated NPC [45]. However, a comprehensive and systemic study of gene expression and epigenetic changes was still lacking; this will be helpful to better understand how cancer evolves and find candidate biomarkers for NPC. 
In our work, through integrating multiple types of datasets using bioinformatics analysis, we identified reliable consensus DEGs. Considering the difference in detectable genes among different platforms, we selected not only recurrent DEGs but also the top genes with markedly differential expression in only one study using the RRA method.

Furthermore, we investigated the interaction between DEGs using differential co-expression analysis, and found that the majority of DCGs were downregulated and tended to lose association in cancer; $98 \%$ of DCPs that lost positive correlations with PAX5 were upregulated, whereas $85 \%$ of DCPs that lost negative correlations were downregulated (Supplementary Figure 1B). This suggested that owing to lost associations, downregulated TSG PAX5 lost regulation of its target genes, and some mis-regulated oncogenes were overexpressed.

NPC is distinguished by differences in immune cell genes, and both tumor genetics and EBV infection influence the tumor microenvironment [3]. Upregulated genes of DNA methyltransferase and PcG proteins were co-expressed with actively expressed EBV genes, especially EBNA1, suggesting EBV interacts with the host genome and affects host genome methylation, thereby causing NPC.

Combining epigenetic data, we found DEGs regulated by aberrant methylation in gene promoter regions or DM miRNAs. 7 downregulated TSGs with aberrant hypermethylation in promoters and 43 upregulated oncogenes regulated by hypermethylated miRNAs were potential biomarkers and therapeutic targets for the precise diagnosis and treatment of NPC. Our work did not analyze survival rates and prognoses owing to the unavailability of clinical data; however, pan-cancer analysis of 14 cancers in TCGA showed that hypermethylated and downregulated MAP4K1, PRKCB, IKZF1, MAP4K1, and $S H I S A 3$ were associated with longer overall survival in HNSC and other cancers. Furthermore, our experimental results validated that $P R K C B$ downregulation in NPC was caused by its promoter hypermethylation. Dysregulated transcription factor $P R K C B$ could be an appropriate target for the development of anticancer drug [46].

\section{Conclusion}

Through comprehensive integrative analysis of different types of data, we have shed more light on the molecular mechanism of carcinogenesis in NPC and provided disease biomarkers for NPC and the hypermethylation of $P R K C B$ could be a novel and promising diagnosis marker.

\section{Abbreviations}

NPC: Nasopharyngeal Carcinoma; EBV: Epstein-Barr virus; DEG: Differentially Expressed Genes; DM: Differentially Methylated; RRA: Robust Rank Aggregation; TSG: Tumor Suppressor Gene; TCGA: The Cancer Genome Atlas; PPI: ProteinProtein Interaction; MTI: miRNA-target interaction; hNEPC: Human nasal epithelial cells; DAC: 5-aza-2'-deoxycytidine.

\section{Supplementary Material}

Supplementary figures.

http://www.jcancer.org/v12p0874s1.pdf

Supplementary table S1-S6.

http://www.jcancer.org/v12p0874s2.xlsx

\section{Acknowledgements}

Thank Dr. Wang Zhen from SIBS for suggestion about gene annotation and interpretation.

\section{Availability of data and materials}

All data generated or analysed during this study are included in this published article and its supplementary information files.

\section{Authors' contributions}

YXL, HL, and YQC conceived this study. YQC and $\mathrm{HL}$ analyzed the data. $\mathrm{CZ}$ and HBL conducted the experiment. All authors participated in writing the manuscript. All authors have read and approved the final manuscript.

\section{Funding}

This work was supported by the National Natural Science Foundation of China (31771472), National Key Research and Development Project (2019YFC1315804, 2016YFC0901704, 2017YFC1201200), Chinese Academy of Sciences (KFJ-STS-QYZD-126, ZDBS-SSW-DQC-02), CAS Youth Innovation Promotion Association, SA-SIBS Scholarship Program, and the Shanghai Municipal Science and Technology Major Project (No.2018SHZDZX01), LCNBI and ZJLab.

\section{Competing Interests}

The authors have declared that no competing interest exists.

\section{References}

1. Wei KR, Zheng RS, Zhang SW, Liang ZH, Li ZM, Chen WQ. Nasopharyngeal carcinoma incidence and mortality in China, 2013. Chinese journal of cancer. 2017; 36: 90.

2. Hildesheim A, Wang CP. Genetic predisposition factors and nasopharyngeal carcinoma risk: a review of epidemiological association studies, 2000-2011: Rosetta Stone for NPC: genetics, viral infection, and other environmental factors. Seminars in cancer biology. 2012; 22: 107-16. 
3. Zhang L, MacIsaac KD, Zhou T, Huang PY, Xin C, Dobson JR, et al. Genomic Analysis of Nasopharyngeal Carcinoma Reveals TME-Based Subtypes. Molecular cancer research : MCR. 2017; 15: 1722-32.

4. Jiang W, Liu N, Chen XZ, Sun Y, Li B, Ren XY, et al. Genome-Wide Identification of a Methylation Gene Panel as a Prognostic Biomarker in Nasopharyngeal Carcinoma. Mol Cancer Ther. 2015; 14: 2864-73.

5. Dai W, Cheung AK, Ko JM, Cheng Y, Zheng H, Ngan RK, et al. Comparative methylome analysis in solid tumors reveals aberrant methylation at chromosome 6p in nasopharyngeal carcinoma. Cancer Med. 2015; 4: 1079-90.

6. Kolde R, Laur S, Adler P, Vilo J. Robust rank aggregation for gene list integration and meta-analysis. Bioinformatics. 2012; 28: 573-80.

7. Team RC. R: A language and environment for statistical computing. 2013.

8. Leek JT, Johnson WE, Parker HS, Jaffe AE, Storey JD. The sva package for removing batch effects and other unwanted variation in high-throughput experiments. Bioinformatics. 2012; 28: 882-3.

9. Ritchie ME, Phipson B, Wu D, Hu Y, Law CW, Shi W, et al. limma powers differential expression analyses for RNA-sequencing and microarray studies. Nucleic acids research. 2015; 43: e47.

10. Love MI, Huber W, Anders S. Moderated estimation of fold change and dispersion for RNA-seq data with DESeq2. Genome biology. 2014; 15: 550.

11. Dobin A, Davis CA, Schlesinger F, Drenkow J, Zaleski C, Jha S, et al. STAR: ultrafast universal RNA-seq aligner. Bioinformatics. 2013; 29: 15-21.

12. Benjamini $Y$, Hochberg YJJotRsss B. Controlling the false discovery rate: a practical and powerful approach to multiple testing. Journal of the Royal Statistical Society. 1995; 57: 289-300.

13. Yang J, Yu H, Liu BH, Zhao Z, Liu L, Ma LX, et al. DCGL v2.0: an R package for unveiling differential regulation from differential co-expression. PloS one. 2013; 8: e79729.

14. KD H. IlluminaHumanMethylation450kanno.ilmn12.hg19: Annotation for Illumina's 450k methylation arrays. 2016; $R$ package version 0.6.0.

15. Yu G, Wang LG, Han Y, He QY. clusterProfiler: an R package for comparing biological themes among gene clusters. OMICS. 2012; 16: 284-7.

16. Lambert SA, Jolma A, Campitelli LF, Das PK, Yin Y, Albu M, et al. The Human Transcription Factors. Cell. 2018; 172: 650-65.

17. Liu Y, Sun J, Zhao M. ONGene: A literature-based database for human oncogenes. J Genet Genomics. 2017; 44: 119-21.

18. Zhao M, Sun J, Zhao Z. TSGene: a web resource for tumor suppressor genes. Nucleic acids research. 2013; 41: D970-6.

19. Gioutlakis A, Klapa MI, Moschonas NK. PICKLE 2.0: A human protein-protein interaction meta-database employing data integration via genetic information ontology. PloS one. 2017; 12: e0186039.

20. Chou $\mathrm{CH}$, Shrestha S, Yang CD, Chang NW, Lin YL, Liao KW, et al. miRTarBase update 2018: a resource for experimentally validated microRNA-target interactions. Nucleic acids research. 2018; 46: D296-D302.

21. Johnson WE, Li C, Rabinovic A. Adjusting batch effects in microarray expression data using empirical Bayes methods. Biostatistics. 2007; 8: 118-27.

22. Zhou F, Gou S, Xiong J, Wu H, Wang C, Liu T. Oncogenicity of LHX2 in pancreatic ductal adenocarcinoma. Mol Biol Rep. 2014; 41: 8163-7.

23. Kuzmanov A, Hopfer U, Marti P, Meyer-Schaller N, Yilmaz M, Christofori G. LIM-homeobox gene 2 promotes tumor growth and metastasis by inducing autocrine and paracrine PDGF-B signaling. Mol Oncol. 2014; 8: 401-16.

24. Xiao Y, Liu J, Peng Y, Xiong X, Huang L, Yang H, et al. GSTA3 Attenuates Renal Interstitial Fibrosis by Inhibiting TGF-Beta-Induced Tubular Epithelial-Mesenchymal Transition and Fibronectin Expression. PloS one. 2016; 11: e0160855.

25. Comamala M, Pinard M, Theriault C, Matte I, Albert A, Boivin M, et al. Downregulation of cell surface CA125/MUC16 induces epithelial-to-mesenchymal transition and restores EGFR signalling in NIH:OVCAR3 ovarian carcinoma cells. Br J Cancer. 2011; 104: 989-99.

26. Liu GJ, Cimmino L, Jude JG, Hu Y, Witkowski MT, McKenzie MD, et al. Pax5 loss imposes a reversible differentiation block in B-progenitor acute lymphoblastic leukemia. Genes Dev. 2014; 28: 1337-50.

27. Lee KY, Geng H, Ng KM, Yu J, van Hasselt A, Cao Y, et al. Epigenetic disruption of interferon-gamma response through silencing the tumor suppressor interferon regulatory factor 8 in nasopharyngeal, esophageal and multiple other carcinomas. Oncogene. 2008; 27: 5267-76.

28. Ren X, Yang X, Cheng B, Chen X, Zhang T, He Q, et al. HOPX hypermethylation promotes metastasis via activating SNAIL transcription in nasopharyngeal carcinoma. Nature communications. 2017; 8: 14053.

29. Zhang J, Li YQ, Guo R, Wang YQ, Zhang PP, Tang XR, et al. Hypermethylation of SHISA3 Promotes Nasopharyngeal Carcinoma Metastasis by Reducing SGSM1 Stability. Cancer Res. 2019; 79: 747-59.

30. Lin YC, Lee YC, Li LH, Cheng CJ, Yang RB. Tumor suppressor SCUBE2 inhibits breast-cancer cell migration and invasion through the reversal of epithelial-mesenchymal transition. J Cell Sci. 2014; 127: 85-100.

31. Payne KJ, Dovat S. Ikaros and tumor suppression in acute lymphoblastic leukemia. Crit Rev Oncog. 2011; 16: 3-12.

32. Chang TC, Wentzel EA, Kent OA, Ramachandran K, Mullendore M, Lee KH, et al. Transactivation of miR-34a by p53 broadly influences gene expression and promotes apoptosis. Mol Cell. 2007; 26: 745-52.

33. Corney DC, Flesken-Nikitin A, Godwin AK, Wang W, Nikitin AY. MicroRNA$34 \mathrm{~b}$ and MicroRNA-34c are targets of p53 and cooperate in control of cell proliferation and adhesion-independent growth. Cancer Res. 2007; 67: 8433-8.
34. Bi WP, Xia M, Wang XJ. miR-137 suppresses proliferation, migration and invasion of colon cancer cell lines by targeting TCF4. Oncol Lett. 2018; 15: 8744-8.

35. Ding F, Zhang S, Gao S, Shang J, Li Y, Cui N, et al. MiR-137 functions as a tumor suppressor in pancreatic cancer by targeting MRGBP. J Cell Biochem. 2018; 119: 4799-807.

36. Zhu X, Li Y, Shen $\mathrm{H}$, Li H, Long $\mathrm{L}$, Hui $\mathrm{L}$, et al, miR-137 inhibits the proliferation of lung cancer cells by targeting Cdc42 and Cdk6. FEBS Lett. 2013; 587: 73-81.

37. Liu X, Li J, Qin F, Dai S. miR-152 as a tumor suppressor microRNA: Target recognition and regulation in cancer. Oncol Lett. 2016; 11: 3911-6.

38. Tang X, Tang J, Liu X, Zeng L, Cheng C, Luo Y, et al. Downregulation of miR-129-2 by promoter hypermethylation regulates breast cancer cell proliferation and apoptosis. Oncol Rep. 2016; 35: 2963-9.

39. Bischoff A, Huck B, Keller B, Strotbek M, Schmid S, Boerries M, et al. miR149 functions as a tumor suppressor by controlling breast epithelial cell migration and invasion. Cancer Res. 2014; 74: 5256-65.

40. Jiang Y, Yan B, Lai W, Shi Y, Xiao D, Jia J, et al. Repression of Hox genes by LMP1 in nasopharyngeal carcinoma and modulation of glycolytic pathway genes by HoxC8. Oncogene. 2015; 34: 6079-91.

41. Jiang C, Wang H, Zhou L, Jiang T, Xu Y, Xia L. MicroRNA-212 inhibits the metastasis of nasopharyngeal carcinoma by targeting SOX4. Oncol Rep. 2017; 38: $82-8$

42. Shi S, Cao X, Gu M, You B, Shan Y, You Y. Upregulated Expression of SOX4 Is Associated with Tumor Growth and Metastasis in Nasopharyngeal Carcinoma. Dis Markers. 2015; 2015: 658141

43. Lin DC, Meng X, Hazawa M, Nagata Y, Varela AM, Xu L, et al. The genomic landscape of nasopharyngeal carcinoma. Nature genetics. 2014; 46: 866-71.

44. Li YY, Chung GT, Lui VW, To KF, Ma BB, Chow C, et al. Exome and genome sequencing of nasopharynx cancer identifies NF-kappaB pathway activating mutations. Nature communications. 2017; 8: 14121

45. Ali SM, Yao M, Yao J, Wang J, Cheng Y, Schrock AB, et al. Comprehensive genomic profiling of different subtypes of nasopharyngeal carcinoma reveals similarities and differences to guide targeted therapy. Cancer. 2017; 123: 3628-37.

46. Darnell JE, Jr. Transcription factors as targets for cancer therapy. Nature reviews Cancer. 2002; 2: 740-9. 